ESAIM: COCV 21 (2015) 301-323

DOI: $10.1051 / \mathrm{cocv} / 2014028$
ESAIM: Control, Optimisation and Calculus of Variations

www.esaim-cocv.org

\title{
CONTROL AND STABILIZATION OF STEADY-STATES IN A FINITE-LENGTH FERROMAGNETIC NANOWIRE
}

\author{
YANnick PRIVAT $^{1}$ AND Emmanuel TrÉlat ${ }^{2}$
}

\begin{abstract}
We consider a finite-length ferromagnetic nanowire, in which the evolution of the magnetization vector is governed by the Landau-Lifshitz equation. We first compute all steady-states of this equation, and prove that they share a quantization property in terms of a certain energy. We study their local stability properties. Then we address the problem of controlling and stabilizing steady-states by means of an external magnetic field induced by a solenoid rolling around the nanowire. We prove that, for a generic placement of the solenoid, any steady-state can be locally exponentially stabilized with a feedback control. Moreover we design this feedback control in an explicit way by considering a finite-dimensional linear control system resulting from a spectral analysis. Finally, we prove that we can steer approximately the system from any steady-state to any other one, provided that they have the same energy level.
\end{abstract}

Mathematics Subject Classification. 58F15, 58F17, 53C35.

Received October 9, 2013. Revised March 27, 2014

Published online January 15, 2015.

\section{INTRODUCTION}

Semiconductor nanowires are emerging as remarkably powerful tools in nanoscience, with the potential of having a significant impact on electronics, but also on numerous other areas of science and technology such as life sciences and healthcare. Nanotechnologies based on semiconductor nanowires promise new generations of devices benefiting from large surface to volume ratios, small active volumes, quantum confinement effects and integration in complex architectures on the nanoscale. Among the applications, magnetic storage on devices such as hard-disks or magnetic MRAMs is one of the most important issues (see, e.g., [24]). Indeed the use of spin injection opens the door towards new spintronic applications and storage technologies while allowing a quick access to information, with a speed which can be millions of times larger than the one in today's hard-disks.

The magnetic moment of a ferromagnetic material represented by a domain $\Omega \subset \mathbb{R}^{3}$ is usually modelled as a time-varying vector field

$$
m: \mathbb{R} \times \Omega \rightarrow \mathcal{S}^{2},
$$

\footnotetext{
Keywords and phrases. Landau-Lifshitz equation, nanowire, control, Kalman condition, feedback stabilization.

1 CNRS, Sorbonne Universités, UPMC Univ Paris 06, UMR 7598, Laboratoire Jacques-Louis Lions, 75005 Paris, France. yannick.privat@upmc.fr

2 Sorbonne Universités, UPMC Univ Paris 06, CNRS UMR 7598, Laboratoire Jacques-Louis Lions, Institut Universitaire de

France, 75005 Paris, France. emmanuel.trelat@upmc.fr
} 
where $\mathcal{S}^{2}$ is the unit sphere of $\mathbb{R}^{3}$, the evolution of which is governed by the Landau-Lifshitz equation (see [20])

$$
\frac{\partial m}{\partial t}=-m \wedge h(m)-m \wedge(m \wedge h(m)),
$$

where the effective field $h(m)$ is defined by

$$
h(m)=2 A \triangle m+h_{d}(m)+h_{\mathrm{ext}} .
$$

The constant $A>0$ is called the exchange constant. By normalization we assume that $A=1 / 2$. The demagnetizing field $h_{d}(m)$ is the solution of the equations $\operatorname{div}\left(h_{d}(m)+m\right)=0$ and $\operatorname{curl}\left(h_{d}(m)\right)=0$ in $\mathcal{D}^{\prime}\left(\mathbb{R}^{3}\right)$, where $m$ is extended to $\mathbb{R}^{3}$ by 0 outside $\Omega$, and $\mathcal{D}^{\prime}\left(\mathbb{R}^{3}\right)$ is the space of distributions on $\mathbb{R}^{3}$. The field $h_{\text {ext }}$ is an external one, for instance it can be an external magnetic field. Other relevant terms may be added for a more accurate physical model, for instance giving an account for the anisotropic behavior of the crystal composing the ferromagnetic material.

The magnetization is usually assumed to satisfy homogeneous Neumann boundary conditions, that is, the normal derivative of $m$ along $\partial \Omega$ vanishes.

The existence of solutions of (1.1) is a challenging issue in general. We refer to $[2,7,21,22,28]$ for results on the existence of global weak solutions or on the existence and uniqueness of local strong solutions. It can be noted that $h(m)=-\nabla E(m)$ with

$$
E(m)=\frac{1}{2} \int_{\Omega}|\nabla m|^{2} \mathrm{~d} x+\frac{1}{2} \int_{\mathbb{R}^{3}}\left|h_{d}(m)\right|^{2} \mathrm{~d} x-\int_{\Omega} h_{\text {ext }} \cdot m \mathrm{~d} x,
$$

which is the energy functional given in [5] in the thermodynamical static model description of ferromagnetic materials. Whereas the Landau-Lifshitz equation (1.1) describes the dynamic evolution in time of the magnetization, the static theory stipulates that the steady-states states of the magnetization field are the minimizers of the energy $E$ : in the ferromagnetic material represented by the domain $\Omega$, there appears a spontaneous magnetization $m$ (of norm 1), minimizing the energy $E(m)$ (see [16]). Note also that, given a solution $m$ of (1.1) with a constant (in time) external field $h_{\text {ext }}$, there holds

$$
\frac{\mathrm{d}}{\mathrm{d} t}(E(m(t, \cdot)))=-\int_{\Omega}|h(m(t, x))-(h(m(t, x)) \cdot m(t, x)) m(t, x)|^{2} \mathrm{~d} x,
$$

and thus this energy functional is naturally nonincreasing along a solution of (1.1).

Since the two terms at the right-hand side of (1.1) are orthogonal, every steady-state of (1.1) must satisfy

$$
m \wedge h(m)=0,
$$

and accordingly the set of steady-states coincides with the set of extremal points of the energy $E$.

The set of steady-states of (1.1) is known to be very rich in the sense that it contains a number of diverse pattern configurations, such as Bloch or Néel walls (see $[13,17]$ ). This diversity could be used in magnetic storage technologies in order to encode information or to perform logic operations (see $[1,4,15,27]$ ).

In this paper we focus on ferromagnetic nanowires with finite length. Such ferromagnetic materials are represented by a domain $\Omega$ which is a truncated cylinder whose ratio $\varepsilon=\frac{\text { radius }}{\text { length }}$ is very small (see Fig. 1). From the mathematical point of view, it has been proved in references $[8,25]$ with $\Gamma$ convergence arguments that, if $\varepsilon$ tends to 0 then one ends up with a one-dimensional model of the ferromagnetic nanowire, with a domain $\Omega=(0, L)$ (here, $L>0$ is the length of the nanowire) and the 1D Landau-Lifshitz equation

$$
\frac{\partial m}{\partial t}=-m \wedge h(m)-m \wedge(m \wedge h(m))
$$




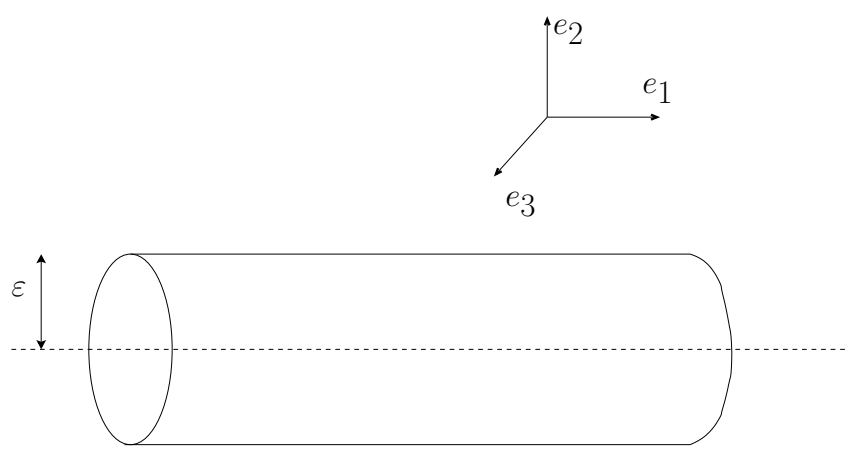

FiguRE 1. Ferromagnetic nanowire.

where $m(t, x) \in \mathcal{S}^{2}$ is the magnetization vector, for every time $t$ and for every $x \in(0, L)$, and where the effective field $h(m)$ takes the more particular form

$$
h(m)=\partial_{x x} m-m_{2} e_{2}-m_{3} e_{3}+h_{\mathrm{ext}} .
$$

The nanowire is here assumed to be parallel to $e_{1}$, the first vector of an orthonormal basis $\left(e_{1}, e_{2}, e_{3}\right)$ of $\mathbb{R}^{3}$, as on Figure 1, and $m=m_{1} e_{1}+m_{2} e_{2}+m_{3} e_{3}$. Morever $m$ satisfies the Neumann boundary conditions

$$
m_{x}(t, 0)=m_{x}(t, L)=0 .
$$

It can be noted that infinite-length $1 \mathrm{D}$ nanowires (that is, $\Omega=\mathbb{R}$ ) have been the subject of many studies in physics or in mathematics. Indeed it is well-known that steady-states of infinite nanowires are Bloch walls, analytically described by usual hyperbolic functions, and whose main physical feature is to induce two almost linear regimes separated by a wall (brutal change of the magnetization). The location of this wall evolves when the nanowire is submitted to an external magnetic field. The dynamics of walls and their stability features have been investigated in many physical studies (such as $[3,4,23,27]$ ), and then has been analyzed mathematically in reference [6] together with control and stabilization properties by means of an external magnetic field in references $[9,10]$.

In this paper we first compute and characterize all steady-states of a finite-length $1 \mathrm{D}$ nanowire. We prove that there exists a finite number of one-parameter families of steady-states that we express analytically in terms of elliptic functions. This finite number increases with the value of the length $L$ of the nanowire. Note that this study of steady-states is similar (but simpler) to the one of [19] where steady-states have been computed for a 1D network of nanowires. Moreover, as in reference [19] we exhibit a quantization property of the steady-states, showing that a certain notion of energy can only take some discrete values. Using spectral tools, we investigate the local stability features of the steady-states.

Then we address the problem of control and stabilization of the steady-states of a finite-length nanowire by means of an external magnetic field. This magnetic field is generated by a solenoid (inductance coil) localized along the nanowire (see Fig. 2). More precisely, assuming that the nanowire is represented by the interval $\Omega=(0, L)$, the solenoid is assumed to generate a magnetic field only along the portion $(a, b)$ of $(0, L)$ (with $0 \leqslant a<b \leqslant L$ arbitrary). From the physical point of view this approximation is acceptable, since outside of the domain of the solenoid the norm of the magnetic field generated by this inductance is rapidly decreasing according to Biot-Savard laws. Moreover, we assume that the axis of the solenoid has an angle of nonzero measure with the axis of the nanowire. Therefore we assume that the magnetic field generated by the solenoid is

$$
h_{\text {ext }}(t)=u(t) \chi_{(a, b)}(x) \vec{d},
$$




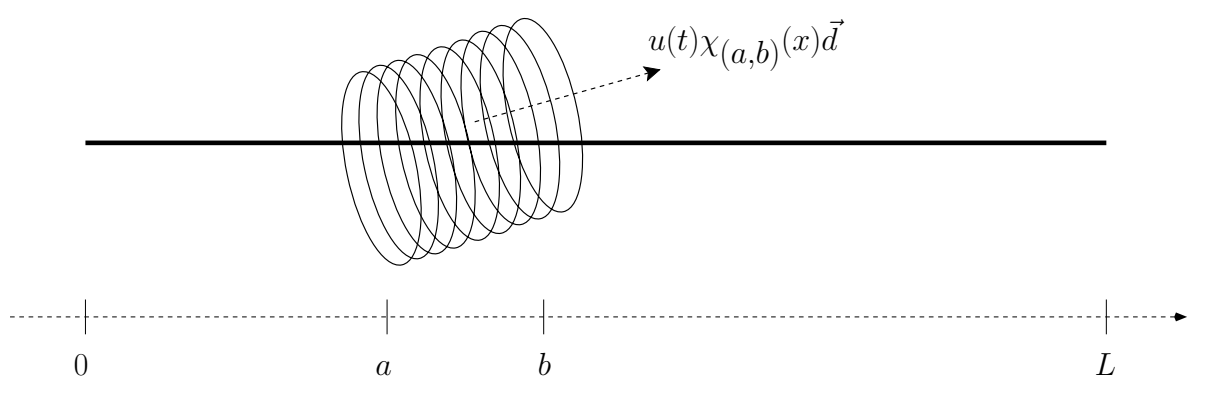

FiguRE 2. The solenoid used as controller.

where $\vec{d}=d_{1} e_{1}+d_{3} e_{3}$ is a fixed vector of $\mathbb{R}^{3}$ with $d_{3} \neq 0$. Here, the notation $\chi_{J}(x)$ stands for the characteristic function of a measurable set $J$, that is, $\chi_{J}(x)=1$ whenever $x \in J$ and $\chi_{J}(x)=0$ otherwise. The scalar $u(t)$ denotes the magnitude at time $t$ of the magnetic field and is our control.

The Landau-Lifshitz equation yields the control system

$$
\begin{aligned}
\frac{\partial m}{\partial t} & =-m \wedge h_{0}(m)-m \wedge\left(m \wedge h_{0}(m)\right)-u \chi_{(a, b)} m \wedge \vec{d}-u \chi_{(a, b)} m \wedge(m \wedge \vec{d}) \\
m_{x}(t, 0) & =m_{x}(t, L)=0
\end{aligned}
$$

with

$$
h_{0}(m)=\partial_{x x} m-m_{2} e_{2}-m_{3} e_{3} .
$$

The problem that we address in the present paper is the following: given two steady-states $\bar{m}^{1}$ and $\bar{m}^{2}$, do there exist a time $T>0$ and a control function $u$ defined on $(0, T)$ such that the corresponding magnetization vector $m$, solution of (1.4) with Neumann boundary conditions (1.5) and starting at $\bar{m}^{1}$, reaches $\bar{m}^{2}$ within time $T$ ? Moreover, can such controls be designed in a nice and robust way?

Moreover we investigate the following practical question: how does the orientation $\vec{d}$ impact the above controllability issue? For instance, can the choice $\vec{d}=e_{1}$ solve the problem?

In the paper we prove that, with a solenoid with $d_{3} \neq 0$ as in Figure 2 and for generic values of $a$ and $b$ that we will characterize in the sequel, any steady-state can be locally exponentially stabilized with an explicit feedback control, which can be moreover designed from a finite-dimensional linear control system. As a second result, we prove that it is possible to steer approximately the magnetization vector from a steady-state to any other one, provided that they have the same level of (quantized) energy. We will actually show that our result holds true for all values of $a$ and $b$ but a finite number of (resonant) values, and hence our result shares some robustness properties.

We stress on the fact that the system is acted upon with a localized magnetic field only (as in Fig. 2), however our results hold in the particular case $a=0, b=L$.

In reference [8], the authors consider a particular steady-state and investigate the case where $d_{3}$, the third direction of the solenoid, is zero. They prove that the resulting control system is stabilizable, but is not asymptotically stabilizable with their feedback law. More details are provided in Remark 3.5. We stress that the main novelty of our paper consists of the asymptotic stabilization results stated in Section 3.1, under generic conditions on the direction of the solenoid. 


\section{QuANTIZED STEADY-STATES AND THEIR STABILITy PROPERTIES}

\subsection{Computation of all steady-states}

Recall that the steady-states of (1.4), in the absence of control $(u=0)$ are characterized by

$$
\begin{array}{ll}
m \wedge h_{0}(m)=0 & x \in(0, L) \\
|m|=1 & x \in(0, L) \\
m_{x}(0)=m_{x}(L)=0, &
\end{array}
$$

where $h_{0}$ is defined by (1.7).

Theorem 2.1. Let $N_{0}=\left[\frac{L}{\pi}\right]$, the integer part of $\frac{L}{\pi}$. There exist $N_{0}$ real numbers $E_{1}, \ldots, E_{N_{0}}$ in $[0,1)$ such that every steady-state of (1.4) is either of the form

$$
\bar{m}(x)=\left(\begin{array}{c} 
\pm 1 \\
0 \\
0
\end{array}\right)= \pm e_{1},
$$

or

$$
\bar{m}(x)=\left(\begin{array}{c}
\cos \theta(x) \\
\cos \omega \sin \theta(x) \\
\sin \omega \sin \theta(x)
\end{array}\right),
$$

for some $\omega \in \mathbb{R}$, where $\theta$ is a solution of the pendulum equation

$$
\begin{aligned}
\theta^{\prime \prime}(x) & =\sin \theta(x) \cos \theta(x), \quad 0<x<L, \\
\theta^{\prime}(0) & =\theta^{\prime}(L)=0,
\end{aligned}
$$

satisfying

$$
\theta^{\prime 2}+\cos ^{2} \theta=\mathrm{Cst}=E_{n} \in\left\{E_{1}, E_{2}, \ldots, E_{N_{0}}\right\} .
$$

In particular the set of steady-states reduces to the two functions given by (2.2) whenever $L<\pi$.

Note that the two steady-states (2.2) can be actually written in the form (2.3) (with $\theta=0$ or $\pi$ ), but with $\theta^{\prime 2}+\cos ^{2} \theta=$ Cst $=1$. We prefer however the presentation above, putting apart the trivial steady-states $(2.2)$.

The steady-states (2.3) consist of $N_{0}$ one-parameter families of steady-states, where the continuous parameter is $\omega \in \mathbb{R}$. They are quantized by the value of what we can call their energy $\theta^{\prime 2}+\cos ^{2} \theta$ (which is constant in $x$ over $(0, L))$ : this energy can only take certain precise values among the set $\left\{E_{1}, E_{2}, \ldots, E_{N_{0}}\right\}$. This quantization of the set of steady-states is due to the Neumann conditions $\theta^{\prime}(0)=\theta^{\prime}(L)=0$, as shown in the proof below.

Note that if $L=\pi$ then there must hold $\theta=\mathrm{Cst}=\pi / 2$. This particular steady-state corresponds to the center of the phase portrait of the pendulum (see Fig. 3).

Proof. Every steady-state of (1.4) can be written as $m=m_{1} e_{1}+m_{2} e_{2}+m_{3} e_{3}$ (function of $x$ only), and (2.1) yields

$$
\begin{array}{cc}
m_{1} m_{3}^{\prime \prime}-m_{1}^{\prime \prime} m_{3}-m_{1} m_{3}=0 & \text { on }(0, L), \\
m_{2} m_{3}^{\prime \prime}-m_{3} m_{2}^{\prime \prime}=0 & \text { on }(0, L), \\
m_{1} m_{2}^{\prime \prime}-m_{1}^{\prime \prime} m_{2}-m_{1} m_{2}=0 & \text { on }(0, L), \\
m_{1}^{2}+m_{2}^{2}+m_{3}^{2}=1 & \text { on }(0, L), \\
m^{\prime}(0)=m^{\prime}(L)=0 . &
\end{array}
$$


The integration of the second equation of (2.5) yields the existence of a real number $\alpha$ such that $m_{2} m_{3}^{\prime}-$ $m_{2}^{\prime} m_{3}=\alpha$ on $(0, L)$. Moreover, since $m$ takes its values in $\mathbb{S}^{2}$, we set

$$
\begin{aligned}
& m_{1}(x)=\cos \theta(x), \\
& m_{2}(x)=\cos \omega(x) \sin \theta(x), \\
& m_{3}(x)=\sin \omega(x) \sin \theta(x),
\end{aligned}
$$

for every $x \in(0, L)$. Then, it follows from $(2.5)$ that

$$
\begin{aligned}
2 \theta^{\prime \prime} \sin \omega+\omega^{\prime \prime} \cos \omega \sin (2 \theta)-\left(\omega^{\prime 2}+1\right) \sin \omega \sin (2 \theta)+4 \omega^{\prime} \theta^{\prime} \cos \omega \cos ^{2} \theta & =0 \\
2 \theta^{\prime \prime} \cos \omega-\omega^{\prime \prime} \sin \omega \sin (2 \theta)-\left(\omega^{\prime 2}+1\right) \cos \omega \sin (2 \theta)-4 \omega^{\prime} \theta^{\prime} \sin \omega \cos ^{2} \theta & =0 \\
\omega^{\prime} \sin ^{2} \theta & =\alpha
\end{aligned}
$$

Moreover, since

$$
\begin{aligned}
m_{1}^{\prime} & =-\theta^{\prime} \sin \theta \\
\left(\begin{array}{l}
m_{2}^{\prime} \\
m_{3}^{\prime}
\end{array}\right) & =\left(\begin{array}{cc}
\cos \omega & -\sin \omega \\
\sin \omega & \cos \omega
\end{array}\right)\left(\begin{array}{l}
\theta^{\prime} \cos \theta \\
\omega^{\prime} \sin \theta
\end{array}\right),
\end{aligned}
$$

the boundary conditions yield $\theta^{\prime}(y) \sin \theta(y)=\theta^{\prime}(y) \cos \theta(y)=\omega^{\prime}(y) \sin \theta(y)=0$ for $y=0$ and $y=L$, hence

$$
\theta^{\prime}(0)=\theta^{\prime}(L)=0 \quad \text { and } \quad \omega^{\prime}(0) \sin \theta(0)=\omega^{\prime}(L) \sin \theta(L)=0
$$

In particular, it follows from (2.13) that necessarily $\alpha=0$ and hence

$$
\omega^{\prime} \sin ^{2} \theta=\text { const. }=0
$$

Then, except the particular cases $\theta=0$ or $\theta=\pi$ (which yield the steady-states (2.2)), we get that $\omega$ is constant.

Now, multiplying (2.11) by $\sin \omega$ and (2.12) by $\cos \omega$ and adding these two equalities, it follows that

$$
\theta^{\prime \prime}=\sin \theta \cos \theta
$$

In particular $\theta^{\prime 2}+\cos ^{2} \theta$ is constant. This is a pendulum equation. Since the solutions $\theta$ of this pendulum equation must satisfy the Neumann conditions $\theta^{\prime}(0)=\theta^{\prime}(L)=0$, they must correspond to pieces of particular closed curves drawn on the phase portrait of Figure 3. More precisely, they must be pieces of periodic solutions, inside the domain enclosed by the separatrices of the pendulum in the phase portrait, with terminal points along the horizontal axis, and with a period $T$ that has to be in a certain ratio with respect to the length $L$ of the nanowire. Indeed the condition $\theta^{\prime}(0)=\theta^{\prime}(L)=0$ imposes that $L$ is an integer multiple of $T / 2$ : there must exist $n \in \mathbb{N}^{*}$ such that

$$
L=n \frac{T}{2}
$$




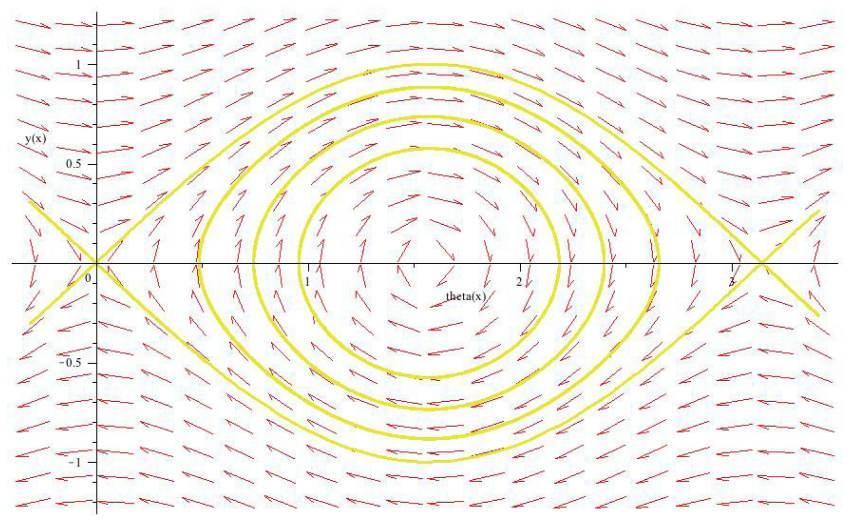

Figure 3. Phase portrait of $(2.4)$ in the plane $\left(\theta, \theta^{\prime}\right)$.

The explicit expression of the solutions of the pendulum equation, as well as their periods, is well-known in function of the elliptic functions ${ }^{3}$. More precisely, one has

$$
\begin{aligned}
\theta^{\prime}(x) & =k \text { cn }\left(x+\operatorname{sn}^{-1}\left(\frac{1}{k} \cos \theta(0), k\right), k\right), \\
\cos \theta(x) & =k \operatorname{sn}\left(x+\operatorname{sn}^{-1}\left(\frac{1}{k} \cos \theta(0), k\right), k\right),
\end{aligned}
$$

for every $x \in(0, L)$, with $\theta^{\prime 2}+\cos ^{2} \theta=k^{2}$ (with $0 \leqslant k<1$, the modulus of the elliptic function). The period of $\theta$ is $T=4 K(k)$. Hence we have obtained that

$$
L=2 n K(k) .
$$

Since $K$ is an increasing function from $[0,1)$ in $[\pi / 2,+\infty)$, the modulus $k$ can take only certain precise values: this equation has exactly $N_{0}$ solutions. The result follows.

Remark 2.2. What is interesting here is the quantization property of the steady-states, whose energy $\theta^{\prime 2}+$ $\cos ^{2} \theta$ can only take certain values. A similar result was obtained in [19] for a network of nanowires. Note that in $[8]$ the authors consider throughout their work the particular steady-state (up to the rotation parameter $\omega$ ) given by (2.3)-(2.4) with $n=1$ (that is, with $\theta$ making half a period in the phase portrait).

\footnotetext{
${ }^{3}$ Recall that, given $k \in(0,1), \tilde{k}=\sqrt{1-k^{2}}$ and $\eta \in[0,1]$, the Jacobi elliptic functions cn, sn and dn are defined from their inverse functions with respect to the first variable,

$$
\begin{aligned}
& \operatorname{cn}^{-1}:(\eta, k) \longmapsto \int_{\eta}^{1} \frac{\mathrm{d} t}{\sqrt{\left(1-t^{2}\right)\left(\tilde{k}^{2}+k^{2} t^{2}\right)}} \\
& \operatorname{sn}^{-1}:(\eta, k) \longmapsto \int_{0}^{\eta} \frac{\mathrm{d} t}{\sqrt{\left(1-t^{2}\right)\left(1-k^{2} t^{2}\right)}} \\
& \operatorname{dn}^{-1}:(\eta, k) \longmapsto \int_{\eta}^{1} \frac{\mathrm{d} t}{\sqrt{\left(1-t^{2}\right)\left(t^{2}+k^{2}-1\right)}}\left(\eta \geqslant \sqrt{1-k^{2}} \text { in that case }\right)
\end{aligned}
$$
}

and the complete integral of the first kind is defined by

$$
K(k)=\int_{0}^{\pi / 2} \frac{d \theta}{\sqrt{1-k^{2} \sin ^{2} \theta}}
$$

The functions $\mathrm{cn}$ and sn are periodic with period $4 K(k)$ while dn is periodic with period $2 K(k)$. 


\subsection{Linearized system around a steady-state}

Let us linearize the control system (1.4) around a steady-state. Let $M_{0}$ be an arbitrary steady-state (we have seen that they exist provided that $L \geqslant \pi$ ). Without loss of generality we assume that $\omega=0$ (since Eq. (1.4) is invariant with respect to rotations in $\omega$ ), and thus that

$$
M_{0}(x)=\left(\begin{array}{c}
\cos \theta(x) \\
\sin \theta(x) \\
0
\end{array}\right)
$$

with $\theta$ solution of the pendulum equation (2.4), and with

$$
\theta^{\prime 2}+\cos ^{2} \theta=E_{n}
$$

for some given $n \in\left\{1, \ldots, N_{0}\right\}$.

Note that the trivial steady-states $(2.2)$ can be put in this form provided that one takes $E_{n}=1$. In what follows, to avoid triviality we assume that the steady-state $M_{0}$ is not of the form (2.2).

Following $[6,8,19]$, we complete $M_{0}$ into the mobile frame $\left(M_{0}(x), M_{1}(x), M_{2}\right)$, with

$$
M_{1}(x)=\left(\begin{array}{c}
-\sin \theta(x) \\
\cos \theta(x) \\
0
\end{array}\right), M_{2}=\left(\begin{array}{l}
0 \\
0 \\
1
\end{array}\right) .
$$

For every solution $m$ of the controlled Landau-Lifshitz equation (1.6), considering $m$ as a perturbation of the steady-state $M_{0}$, since $|m(t, x)|=1$ pointwisely, we decompose $m: \mathbb{R}_{+} \times \mathbb{R} \longrightarrow \mathbb{S}^{2} \subset \mathbb{R}^{3}$ in the mobile frame as

$$
m(t, x)=\sqrt{1-r_{1}^{2}(t, x)-r_{2}^{2}(t, x)} M_{0}(x)+r_{1}(t, x) M_{1}(x)+r_{2}(t, x) M_{2} .
$$

According to ([8], Sect. 4) and ([19], Sect. 3.1), $m$ is solution of (1.4) if and only if $r=\left(\begin{array}{l}r_{1} \\ r_{2}\end{array}\right)$ satisfies

$$
\frac{\partial r}{\partial t}=\mathcal{A} r+B u+R\left(x, r, r_{x}, r_{x x}, u\right)
$$

where

$$
\mathcal{A}=\left(\begin{array}{cc}
A+\mathrm{Id} & A+E_{n} \mathrm{Id} \\
-(A+\mathrm{Id}) & A+E_{n} \mathrm{Id}
\end{array}\right)
$$

with

$$
A=\partial_{x x}^{2}-2 \cos ^{2} \theta \mathrm{Id}
$$

defined on the domain

$$
D(A)=\left\{f \in H^{2}(0, L) \mid f^{\prime}(0)=f^{\prime}(L)=0\right\}
$$

(and hence $D(\mathcal{A})=D(A) \times D(A)$ ), where

$$
B(x)=\chi_{(a, b)}(x)\left(\begin{array}{l}
d_{3}-d_{1} \sin \theta(x) \\
d_{3}+d_{1} \sin \theta(x)
\end{array}\right),
$$

and where the higher-order terms are

$$
R\left(x, r, r_{x}, r_{x x}, u\right)=G(r) r_{x x}+H_{1}(x, r) r_{x}+H_{2}(r)\left(r_{x}, r_{x}\right)+P(x, r, u),
$$


where

$$
\begin{gathered}
G(r)=\left(\begin{array}{cc}
\frac{r_{1} r_{2}}{\sqrt{1-|r|^{2}}} & \frac{r_{2}^{2}}{\sqrt{1-|r|^{2}}}+\sqrt{1-|r|^{2}}-1 \\
-\frac{r_{1}^{2}}{\sqrt{1-|r|^{2}}}-\sqrt{1-|r|^{2}}+1 & -\frac{r_{1} r_{2}}{\sqrt{1-|r|^{2}}}
\end{array}\right), \\
H_{1}(x, r)=\frac{2 \theta^{\prime}(x)}{\sqrt{1-|r|^{2}}}\left(\begin{array}{cc}
r_{2} \sqrt{1-|r|^{2}}-r_{1} r_{2}^{2} & -r_{2}\left(1-r_{1}^{2}\right) \\
r_{2}\left(1-r_{2}^{2}\right) & \sqrt{1-|r|^{2}} r_{2}+r_{1} r_{2}^{2}
\end{array}\right),
\end{gathered}
$$

$H_{2}(r)$ is the quadratic form on $\mathbb{R}^{2}$ defined by

$$
H_{2}(r)(X, X)=\frac{\left(1-|r|^{2}\right) X^{\top} X+\left(r^{\top} X\right)^{2}}{(1-|r|)^{3 / 2}}\left(\begin{array}{c}
\sqrt{1-|r|^{2}} r_{1}+r_{2} \\
\sqrt{1-|r|^{2}} r_{2}-r_{1}
\end{array}\right),
$$

and

$$
P(x, r, u)=\left(\begin{array}{l}
P_{1}(x, r, u) \\
P_{2}(x, r, u)
\end{array}\right)
$$

with

$$
\begin{aligned}
P_{1}(x, r, u)= & -r_{1} \sqrt{1-|r|^{2}} \cos \theta+\left(r_{1}^{2}-r_{2}\right) \sin \theta \\
& -u \chi_{[a, b]} d_{1}\left(\left(r_{2}+r_{1} \sqrt{1-|r|^{2}}\right) \cos \theta-r_{1}^{2} \sin \theta\right) \\
& -u \chi_{[a, b]} d_{3}\left(1-\sqrt{1-|r|^{2}}+r_{1} r_{2}\right)
\end{aligned}
$$

and

$$
\begin{aligned}
P_{2}(x, r, u)= & \left(r_{1}-r_{2} \sqrt{1-|r|^{2}}\right) \cos \theta+\sin \theta\left(r_{1} r_{2}+\sqrt{1-|r|^{2}}-1\right) \\
& -u \chi_{[a, b]} d_{1}\left(\left(1-\sqrt{1-|r|^{2}}-r_{1} r_{2}\right) \sin \theta+\left(r_{2} \sqrt{1-|r|^{2}}-r_{1}\right) \cos \theta\right) \\
& -u \chi_{[a, b]} d_{3} r_{2}^{2} .
\end{aligned}
$$

What is important is the estimates

$$
G(r)=O\left(|r|^{2}\right), \quad H_{1}(r)=O(|r|), \quad H_{2}(r)=O(|r|),
$$

and in particular the fact that there exists a constant $C>0$ such that, if $|r|^{2} \leqslant \frac{1}{2}$, then

$$
|R(x, r, p, q, u)| \leqslant C\left(|r|^{2}|q|+|r||p|+|r||p|^{2}+|u||r|\right),
$$

for every $x \in \mathbb{R},(r, p, q) \in\left(\mathbb{R}^{2}\right)^{3}$, and $u \in \mathbb{R}^{2}$. This estimate shows that $R\left(x, r, r_{x}, r_{x x}, u\right)$ is a term of higher order in (2.19) (remainder term).

\subsection{Spectral properties of the steady-states}

In this section we analyze the spectral properties of the steady-states of the uncontrolled Landau-Lifshitz equation, that is, we take $u(\cdot)=0$ in (2.19), and we analyze the underlying linear operator $\mathcal{A}$ defined by $(2.20)$.

As we will see, $\mathcal{A}$ is not diagonalizable, however it can be diagonalized by blocks. We first need to analyze the spectral properties of the operator $A$ defined by (2.21). Note that

$$
\mathcal{A} r=\mathcal{A}\left(\begin{array}{l}
r_{1} \\
r_{2}
\end{array}\right)=\left(\begin{array}{r}
(A+\mathrm{Id}) r_{1}+\left(A+E_{n} \mathrm{Id}\right) r_{2} \\
-(A+\mathrm{Id}) r_{1}+\left(A+E_{n} \mathrm{Id}\right) r_{2}
\end{array}\right),
$$

for every $r \in D(\mathcal{A})=D(A) \times D(A)$. 
Spectral analysis of $A$. The operator $A=\partial_{x x}^{2}-2 \cos ^{2} \theta$ Id defined on the domain $D(A)=\{f \in$ $\left.H^{2}(0, L) \mid f^{\prime}(0)=f^{\prime}(L)=0\right\}$ is clearly self-adjoint in $L^{2}(0, L)$. As a first preliminary remark, we see using an integration by parts that

$$
\left\langle\left(A+E_{n} \mathrm{Id}\right) f, f\right\rangle_{L^{2}}=-\left\|f^{\prime}-\theta^{\prime} \operatorname{cotan} \theta f\right\|_{L^{2}}^{2},
$$

for every $f \in D(A)$. Therefore,

$$
\left\langle\left(A+E_{n} \mathrm{Id}\right) f, f\right\rangle_{L^{2}} \leqslant 0,
$$

which means that the operator $A+E_{n}$ Id defined on $D(A)$ is nonpositive.

As a second preliminary remark, we are able to compute two eigenfunctions of $A$. Indeed, we have

$$
A \sin \theta=-E_{n} \sin \theta
$$

and that

$$
A \cos \theta=-\left(1+E_{n}\right) \cos \theta .
$$

Note that, if $E_{n}=0$ then $\theta=\pi / 2$ and in that case $\cos \theta$ is not an eigenfunction. Similarly, if $E_{n}=1$ then $\theta=0$ or $\pi$ and in that case $\sin \theta$ is not an eigenfunction. For all other cases, one has $0<E_{n}<1$, and we have thus computed two eigenfunctions. Moreover, since $A+E_{n}$ Id is nonpositive, it follows that $-E_{n}$ is the largest eigenvalue of $A$, associated with the eigenfunction $\sin \theta$.

Proposition 2.3. There exists a Hilbert basis $\left(e_{j}\right)_{j \in \mathbb{N}}$ of $L^{2}(0, L)$, consisting of eigenfunctions of $A$, associated with real eigenvalues $\lambda_{j}$ that are simple, with

$$
-\infty<\cdots<\lambda_{j}<\cdots<\lambda_{1}<\lambda_{0}=-E_{n}
$$

and $\lambda_{j} \rightarrow-\infty$ as $j \rightarrow+\infty$. Moreover,

- the largest eigenvalue $\lambda_{0}=-E_{n}$ is associated with the eigenfunction $e_{0}=\sin \theta$;

- the eigenfunction $e_{j}$ vanishes exactly $j$ times on $(0, L)$;

- the $(n+1)$ th eigenvalue is $\lambda_{n}=-\left(1+E_{n}\right)$ and is associated with the eigenfunction $e_{n}=\cos \theta$;

- -1 is not an eigenvalue of the operator $A$.

Remark 2.4. The fact that -1 is not an eigenvalue of the operator $A$ will play an important role in the sequel (see Sect. 3). It will ensure in particular that our controllability results hold true by using only one single solenoid instead of two or more.

Proof. The existence of the Hilbert basis of eigenfunctions follows from the application of the spectral theorem to the compact self-adjoint operator $f \in L^{2}(0, L) \mapsto w \in L^{2}(0, L)$, where $w$ is the unique solution of

$$
\begin{aligned}
& w^{\prime \prime}-\left(2 \cos ^{2} \theta+1\right) w=f, x \in(0, L) \\
& w^{\prime}(0)=w^{\prime}(L)=0 .
\end{aligned}
$$

The simplicity of the eigenvalues of $A$ and the nodal domain property are standard results for Sturm-Liouville operators with Neumann boundary conditions (see for example [14,29]). Since $A \cos \theta=-\left(1+E_{n}\right) \cos \theta$ and since the function $\cos \theta$ vanishes $n$ times along $(0, L)$, we deduce that $-\left(1+E_{n}\right)$ is the $(n+1)$ th eigenvalue of $A$.

It remains now to prove that -1 is not an eigenvalue of $A$. We define the functions $F_{1}$ and $F_{2, \gamma}$ by

$$
F_{1}(x)=\theta^{\prime}(x) \quad \text { and } \quad F_{2, \gamma}(x)=\theta^{\prime}(x) \int_{\gamma}^{x} \frac{\mathrm{d} t}{\theta^{\prime}(t)^{2}},
$$


where $\gamma$ denotes any real number that does not belong to the set of zeros of the function $\theta^{\prime}$. Denote by $x_{0}$ any zero of the function $\theta^{\prime}$. Notice that, since $\theta$ satisfies $(2.4)$, one has $\theta^{(3)}\left(x_{0}\right)=0$. Using a Taylor expansion with integral rest, it follows that there exist two functions $\eta_{1}$ and $\eta_{2}$, respectively smooth in $(0, L)$ and smooth at $x_{0}$, such that $\eta_{1}\left(x_{0}\right)=\eta_{2}\left(x_{0}\right)=0$ and

$$
\begin{aligned}
\theta^{\prime}(x) & =\theta^{\prime \prime}\left(x_{0}\right)\left(x-x_{0}\right)+\left(x-x_{0}\right)^{2} \eta_{1}(x) \\
\frac{1}{\theta^{\prime}(x)^{2}} & =\frac{1}{\theta^{\prime \prime}\left(x_{0}\right)^{2}\left(x-x_{0}\right)^{2}}+\eta_{2}(x) .
\end{aligned}
$$

Moreover, the function $\eta_{2}$ is smooth at $x_{0}$ and every point where $\theta^{\prime}$ does not vanish. According to the expansions of $\theta^{\prime}$ and $\frac{1}{\theta^{\prime 2}}$ above, one easily computes

$$
F_{2, \gamma}(x)=-\frac{1}{\theta^{\prime \prime}\left(x_{0}\right)}+\frac{x-x_{0}}{\theta^{\prime \prime}\left(x_{0}\right)\left(\alpha-x_{0}\right)}+\underset{x \rightarrow x_{0}}{\mathrm{o}}\left(x-x_{0}\right),
$$

showing in particular that

$$
F_{2, \gamma}\left(x_{0}\right)=-\frac{1}{\theta^{\prime \prime}\left(x_{0}\right)} \quad \text { and } \quad \frac{d F_{2, \gamma}}{\mathrm{d} x}\left(x_{0}\right)=\frac{1}{\theta^{\prime \prime}\left(x_{0}\right)\left(\alpha-x_{0}\right)} .
$$

Moreover the space of solutions of the ordinary differential equation

$$
w^{\prime \prime}-2 \cos ^{2} \theta w=-w \text { in }(0, L)
$$

is exactly $\operatorname{Span}\left\{F_{1}, F_{2, \gamma}\right\}$. It follows that -1 is an eigenvalue of $A$ if, and only if there exists $\gamma \in \mathbb{R}$ such that the function $y: x \mapsto F_{2, \gamma}^{\prime}(0) F_{1}(x)-F_{1}^{\prime}(0) F_{2, \gamma}(x)$ satisfies $y^{\prime}(L)=0$. This is equivalent to the condition

$$
\frac{F_{1}^{\prime}(0)}{F_{1}^{\prime}(L)}=\frac{F_{2, \gamma}^{\prime}(0)}{F_{2, \gamma}^{\prime}(L)}
$$

Noting that $\frac{F_{1}^{\prime}(0)}{F_{1}^{\prime}(L)}=\frac{\theta^{\prime \prime}(0)}{\theta^{\prime \prime}(L)}$ and $\frac{F_{2, \gamma}^{\prime}(0)}{F_{2, \gamma}^{\prime}(L)}=\frac{\gamma-L}{\gamma} \frac{\theta^{\prime \prime}(L)}{\theta^{\prime \prime}(0)}$ according to the previous computations, and using that $\theta^{\prime \prime}(0)^{2}=\theta^{\prime \prime}(L)^{2}$, the condition $(2.25)$ rewrites $\frac{\gamma-L}{\gamma}=1$, which is not satisfied. The conclusion follows.

Hence, at this step, we have the following array, summarizing the spectral properties of $A$ :

$$
\begin{aligned}
& A e_{0}=\lambda_{0} e_{0}, \quad e_{0}=\sin \theta, \quad \lambda_{0}=-E_{n} \\
& \begin{array}{ccc}
\vdots & \vdots & \vdots \\
A e_{n}=\lambda_{n} e_{n}, & e_{n}=\cos \theta, & \lambda_{n}=-1-E_{n}
\end{array} \\
& \begin{array}{ccr}
\vdots & \vdots & \\
A e_{j}=\lambda_{j} e_{j}, & & \lambda_{j} \\
\vdots & \vdots & \downarrow
\end{array}
\end{aligned}
$$

Let us now perform the spectral analysis of the operator $\mathcal{A}$ defined by (2.20).

Spectral analysis of $\mathcal{A}$. For every $r=\left(\begin{array}{l}r_{1} \\ r_{2}\end{array}\right) \in D(\mathcal{A})=D(A) \times D(A)$, one has, from Proposition 2.3,

$$
r_{1}=\sum_{j=0}^{+\infty} r_{1 j} e_{j}, \quad r_{2}=\sum_{k=0}^{+\infty} r_{2 k} e_{k} .
$$


Hence

$$
r=\left(\begin{array}{l}
\sum_{j=0}^{+\infty} r_{1 j} e_{j} \\
\sum_{k=0}^{+\infty} r_{2 k} e_{k}
\end{array}\right)=\sum_{j=0}^{+\infty} r_{1 j}\left(\begin{array}{c}
e_{j} \\
0
\end{array}\right)+\sum_{k=0}^{+\infty} r_{2 k}\left(\begin{array}{c}
0 \\
e_{k}
\end{array}\right)=\sum_{j=0}^{+\infty} r_{j} \tilde{e}_{j}
$$

where $\left(\tilde{e}_{j}\right)_{j \in \mathbb{N}}$ is the orthonormal basis of $L^{2}\left(0, L ; \mathbb{R}^{2}\right)$ built from the orthonormal basis $\left(e_{j}\right)_{j \in \mathbb{N}}$ of $L^{2}(0, L)$ by the usual diagonal procedure used to establish the countability of $\mathbb{N}^{2}$, that is:

$$
\tilde{e}_{2 j}=\left(\begin{array}{c}
e_{j} \\
0
\end{array}\right), \quad \tilde{e}_{2 j+1}=\left(\begin{array}{c}
0 \\
e_{j}
\end{array}\right),
$$

for every $j \in \mathbb{N}$. Here, we have set

$$
r_{j}=\left\langle r, \tilde{e}_{j}\right\rangle_{L^{2}\left(0, L ; \mathbb{R}^{2}\right)}
$$

for every $j \in \mathbb{N}$. It can be noted that

$$
\begin{aligned}
r_{2 j} & =\left\langle r, \tilde{e}_{2 j}\right\rangle_{L^{2}\left(0, L ; \mathbb{R}^{2}\right)}=\left\langle r_{1}, e_{j}\right\rangle_{L^{2}(0, L)}=r_{1 j}, \\
r_{2 j+1} & =\left\langle r, \tilde{e}_{2 j+1}\right\rangle_{L^{2}\left(0, L ; \mathbb{R}^{2}\right)}=\left\langle r_{2}, e_{j}\right\rangle_{L^{2}(0, L)}=r_{2 j},
\end{aligned}
$$

for every $j \in \mathbb{N}$. Moreover, we have

$$
\mathcal{A} \tilde{e}_{2 j}=\left(\begin{array}{r}
(A+\mathrm{Id}) A+E_{n} \mathrm{Id} \\
-(A+\mathrm{Id}) A+E_{n} \mathrm{Id}
\end{array}\right)\left(\begin{array}{c}
e_{j} \\
0
\end{array}\right)=\left(\begin{array}{r}
\left(\lambda_{j}+1\right) e_{j} \\
-\left(\lambda_{j}+1\right) e_{j}
\end{array}\right)=\left(\lambda_{j}+1\right) \tilde{e}_{2 j}-\left(\lambda_{j}+1\right) \tilde{e}_{2 j+1},
$$

and

$$
\mathcal{A} \tilde{e}_{2 j+1}=\left(\begin{array}{r}
(A+\mathrm{Id}) A+E_{n} \mathrm{Id} \\
-(A+\mathrm{Id}) A+E_{n} \mathrm{Id}
\end{array}\right)\left(\begin{array}{c}
0 \\
e_{j}
\end{array}\right)=\left(\begin{array}{c}
\left(\lambda_{j}+E_{n}\right) e_{j} \\
\left(\lambda_{j}+E_{n}\right) e_{j}
\end{array}\right)=\left(\lambda_{j}+E_{n}\right) \tilde{e}_{2 j}+\left(\lambda_{j}+E_{n}\right) \tilde{e}_{2 j+1},
$$

for every $j \in \mathbb{N}$. In other words, the operator $\mathcal{A}$ restricted to the two-dimensional space $\operatorname{Span}\left(\tilde{e}_{2 j}, \tilde{e}_{2 j+1}\right)$ is represented by the $2 \times 2$ matrix

$$
A_{j}=\left(\begin{array}{cc}
\lambda_{j}+1 & \lambda_{j}+E_{n} \\
-\left(\lambda_{j}+1\right) & \lambda_{j}+E_{n}
\end{array}\right)
$$

in the basis $\left(\tilde{e}_{2 j}, \tilde{e}_{2 j+1}\right)$.

We have thus obtained the following corollary.

Corollary 2.5. The operator $\mathcal{A}$ is diagonalizable in $2 \times 2$ blocks. More precisely in the orthonormal basis $\left(\tilde{e}_{j}\right)_{j \in \mathbb{N}}$ the operator $\mathcal{A}$ is represented by the infinite-dimensional matrix

$$
\left(\begin{array}{ccccc}
A_{0} & 0 & \cdots & & \\
0 & A_{1} & \ddots & & \\
0 & 0 & \ddots & & \\
\vdots & & \ddots & A_{j} & \\
& & & & \ddots
\end{array}\right)
$$

which is diagonal by $2 \times 2$ blocks $A_{j}$, where $A_{j}$ is defined by $(2.26)$.

Hence, in order to analyze the local stability of the steady-state $M_{0}$ it suffices to analyze the spectrum of $A_{j}$. We have the following immediate lemma. 
Lemma 2.6. The matrix $A_{j}$ is Hurwitz if and only if $\lambda_{j}<-1$.

Recall that a matrix is said to be Hurwitz if all its eigenvalues have a negative real part.

Since $\lambda_{0}=-E_{n}$ and $\lambda_{j}<-1$ for every $j \geqslant n$, the following result follows (note here the importance of the fact that -1 is not an eigenvalues of $A$, since the value -1 is a pivot value in the stability properties).

Corollary 2.7. Only the two trivial steady-states (2.2) are stable. Any other steady-state (2.3) is unstable for the uncontrolled Landau-Lifschitz equation (1.6) (that is, with $u=0$ ). More precisely, in the spectral expansion of Corollary 2.5, at most the $2 n$ first equations are unstable and all others are locally asymptotically stable.

Remark 2.8. Actually the two trivial steady-states (2.2) are even globally stable since they make vanish the energy defined by (1.2), which besides is nonincreasing according to (1.3).

\section{Control and stabilization Results}

\subsection{The main results}

Theorem 3.1. If $d_{3} \neq 0$ then every steady-state is locally exponentially stabilizable in $H^{1}$ topology, for all values of $0 \leqslant a<b \leqslant L$ but a finite number of choices, by means of feedback controls that can be designed by usual pole-shifting from a finite-dimensional linear control system.

More precisely, let $a \in(0, L)$ arbitrary and let $\vec{d}=\left(d_{1}, d_{2}, d_{3}\right)^{\top} \in \mathbb{R}^{3}$ be such that $d_{3} \neq 0$. There exists a finite discrete set $\mathcal{Z} \subset(0, L)$ such that, for every $b$ in $(0, L) \backslash \mathcal{Z}$ such that $a<b$, there exist a neighborhood $\mathcal{W}$ of $\bar{m}$ in $H^{1}\left(0, L ; \mathbb{R}^{3}\right)$ and a smooth feedback control function $u$ such that every solution $m^{\delta}$ of (1.6) associated with this control, with initial condition in $\mathcal{W}$, converges exponentially to $\bar{m}$ in $H^{1}$ topology.

The strategy of the proof is the following. We consider the linearization of (1.6) around a steady-state, which is given by (2.19). Using the spectral decomposition of (2.19) into an infinite number of two-dimensional systems, we isolate the $n$ first $(2 \times 2$ blocks $)$ modes, which are known to contain all unstable modes, whereas all other ones are naturally stable. On the finite-dimensional linear control system containing all unstable modes, we verify that, under a generic condition on the lengths of the solenoid, the Kalman condition holds true. Then using the pole-shifting theorem we design an explicit feedback control stabilizing exponentially this finite-dimensional system, as well as an explicit Lyapunov function. Finally, we prove that this control stabilizes exponentially as well the whole infinite-dimensional system, by constructing an appropriate Lyapunov function. Note that this last part is nontrivial since the feedback controls built on the finite-dimensional subsystem could destabilize some stable modes of the other infinite-dimensional part (also, the remainder terms have to be taken into account), and the design of an appropriate Lyapunov function requires some care.

Remark 3.2. It can be noted that, with a little additional effort in the proof, one can establish a stabilization result in any topology $H^{s}$ with $s>1$.

Remark 3.3. It can be noticed that the feedback control $u$ exhibited in the proof of Theorem 3.1 does not require the knowledge of the complete solution $(t, x) \mapsto m(t, x)$ of the system (1.6), but only the knowledge of a finite number of integral quantities of the kind $\int_{a}^{b} m(t, x) e_{j}(x) \mathrm{d} x$, where $\left(e_{j}\right)_{j \in \mathbb{N}}$ denotes the Hilbert basis introduced in Proposition 2.3. This is physically more relevant, and the quantities that have to be measured are moments of the magnetization field.

Remark 3.4 (on the robustness of the control). As noted in Remark 3.3, the control law we exhibit in the proof of Theorem 3.1 is constructed from the knowledge of several integral quantities. Practically speaking, these quantities are evaluated with the help of measurements which may be noised. However, since the strategy employed here is based on the use of a Lyapunov function, it is actually robust. Moreover, the number of critical values of the real number $b$ for which the conclusion of this theorem does not hold true anymore is finite. It allows to claim that the sensitivity of the control method presented in the proof of Theorem 3.1 with respect to all the parameters is minimal. 
Remark 3.5. It is important to stress that our result (asymptotic stabilization with a linear feedback) cannot be established with a solenoid whose axis is parallel to the nanowire (that is, with $d_{3}=0$ ). We will indeed prove further that the Kalman condition is then never satisfied for the unstable finite-dimensional part of the system.

In [8] the authors consider the particular steady-state $M_{0}$ with $n=1$ (that is, with $\theta$ making half a period in the phase portrait), with $a=0, b=L$ and $d_{3}=0$, that is, with a coil rolling around the whole nanowire and having the same axis. They provide an explicit linear feedback control such that the closed-loop system is stable (but not asymptotically stable). More precisely, all local variables of the system around the steady-state converge locally exponentially, except the variable $\omega$ describing the rotation of the magnetization vector, which is stable only.

For $n>1$ and $d_{3}=0$ it is not possible to stabilize asymptotically a nontrivial steady-state with a linear feedback (since the Kalman condition is not satisfied) but the question is open to stabilize it with a nonlinear one, or to design a linear feedback making the closed-loop system stable as in [8].

The next result shows that it is always possible to steer the system from a steady-state to any other one, provided that they have the same level of energy $\theta^{\prime 2}+\cos ^{2} \theta=E_{n}$.

Theorem 3.6. Under the generic assumptions of Theorem 3.1, given two steady-states

$$
\bar{m}^{1}(x)=\left(\begin{array}{c}
\cos \theta(x) \\
\cos \omega_{1} \sin \theta(x) \\
\sin \omega_{1} \sin \theta(x)
\end{array}\right), \quad \bar{m}^{2}(x)=\left(\begin{array}{c}
\cos \theta(x) \\
\cos \omega_{2} \sin \theta(x) \\
\sin \omega_{2} \sin \theta(x)
\end{array}\right),
$$

with $\omega_{1} \in \mathbb{R}, \omega_{2} \in \mathbb{R}$, for every $\varepsilon>0$ there exist a time $T>0$ and a controls $u \in L^{2}(0, T)$ such that every solution $m$ of (1.6) satisfying

$$
\left\|m(0, \cdot)-\bar{m}^{1}\right\|_{H^{1}\left(0, L ; \mathbf{R}^{3}\right)} \leqslant \varepsilon
$$

satisfies at time $T$

$$
\left\|m(T, \cdot)-\bar{m}^{2}\right\|_{H^{1}\left(0, L ; \mathbf{R}^{3}\right)} \leqslant \varepsilon .
$$

Moreover this can be done with an explicit smooth feedback control that is built from a finite-dimensional linear control system.

The strategy of the proof consists in stabilizing the system along a path of steady-states joining $\bar{m}^{1}$ and $\bar{m}^{2}$. Such a path exists whenever the two steady-states have the same level of energy. This strategy is inspired by $[11,12]$ where it has been used in order to control semilinear heat and wave equations to steady-states.

Note that, of course, the approximate controllability result (in large time) claimed in Theorem 3.6 follows by using repeatedly Theorem 3.1, by jumping from one neighborhood to the other along a path of steady-states. The strategy that we propose is however more direct and effective and consists of stabilizing a slowly-varying in time finite-dimensional linear control system.

An interesting open question is to know whether or not it is possible to steer the system from a steady-state to any other one, not necessarily having the same level of energy. The question is probably difficult and does not seem to be solvable with the above strategy, due to the fact that there does not seem to exist any path of generalized steady-states (that is, steady-states with nonzero constant controls) joining $\bar{m}^{1}$ and $\bar{m}^{2}$.

Another interesting issue is to determine, whenever it exists, the location of the solenoid used as control ensuring optimal controllability properties.

\subsection{Proof of the results}

Let us first prove Theorem 3.1. Our objective is to design an explicit feedback control locally stabilizing the system (1.4) around a given steady-state (2.3) (note that the trivial steady-states (2.2) are stable so there is nothing to do).

We follow the steps described in the above strategy. 
Consider a steady-state, assumed to be $M_{0}$ without loss of generality, as in Section 2.2. Then, locally around $M_{0}$, every solution $m$ can be written as (2.18) in the frame $\left(M_{0}, M_{1}, M_{2}\right)$, and the Landau-Lifshitz equation is then locally equivalent to (2.19).

Linearized system and spectral expansions. First of all, we consider the linearized system around $M_{0}$, that is (2.19) without the remainder term $R\left(x, r, r_{x}, r_{x x}, u\right)$, which is

$$
\frac{\partial r}{\partial t}=\mathcal{A} r+B u
$$

with $\mathcal{A}$ defined by (2.20) and $B$ defined by (2.22). Using the spectral expansions developed in Section 2.3, and following in particular the analysis made to derive Corollary 2.5, this system is equivalent to the series of linear autonomous control systems

$$
\frac{\mathrm{d}}{\mathrm{d} t}\left(\begin{array}{l}
r_{1 j}(t) \\
r_{2 j}(t)
\end{array}\right)=A_{k}\left(\begin{array}{l}
r_{1 j}(t) \\
r_{2 j}(t)
\end{array}\right)+B_{j} u(t), \quad k \in \mathbb{N}
$$

(note that $r_{1 j}(t)=r_{2 j}(t)$ and $\left.r_{2 j}(t)=r_{2 j+1}(t)\right)$, with

$$
A_{j}=\left(\begin{array}{cc}
\lambda_{j}+1 & \lambda_{j}+E_{n} \\
-\left(\lambda_{j}+1\right) & \lambda_{j}+E_{n}
\end{array}\right), \quad B_{j}=\left(\begin{array}{c}
\int_{a}^{b}\left(d_{3}-d_{1} \sin \theta(x)\right) e_{j}(x) \mathrm{d} x \\
\int_{a}^{b}\left(d_{3}+d_{1} \sin \theta(x)\right) e_{j}(x) \mathrm{d} x
\end{array}\right) .
$$

As explained above, we first focus on the $n$ first systems of (3.2), which contain all unstable modes according to Corollary 2.7, and which can be written as the finite-dimensional linear autonomous control system in $\mathbb{R}^{2 n}$

$$
\dot{r}_{u n}(t)=A_{u n} r_{u n}(t)+B_{u n} u(t),
$$

with

and

$$
r_{u n}(t)=\left(\begin{array}{c}
r_{10}(t) \\
r_{20}(t) \\
\vdots \\
r_{1 n-1}(t) \\
r_{2 n-1}(t)
\end{array}\right)=\left(\begin{array}{c}
r_{0}(t) \\
r_{1}(t) \\
\vdots \\
r_{2 n-2}(t) \\
r_{2 n-1}(t)
\end{array}\right)
$$

$$
A_{u n}=\left(\begin{array}{cccc}
A_{0} & 0 & \cdots & 0 \\
0 & A_{1} & \ddots & \vdots \\
\vdots & \ddots & \ddots & 0 \\
0 & \cdots & 0 & A_{n-1}
\end{array}\right) \text { and } B_{u n}=\left(\begin{array}{c}
B_{0} \\
B_{1} \\
\vdots \\
B_{n-1}
\end{array}\right) \text {. }
$$

This is the first, finite-dimensional part, of the complete system (3.2), whereas the second (infinitedimensional) part of it, containing only stable modes, is written as

$$
\dot{r}_{s t}(t)=A_{s t} r_{s t}(t)+B_{s t} u(t),
$$

with

$$
r_{s t}(t)=\left(\begin{array}{c}
r_{1 n}(t) \\
r_{2 n}(t) \\
\vdots \\
r_{1 j}(t) \\
r_{2 j}(t) \\
\vdots
\end{array}\right)=\left(\begin{array}{c}
r_{2 n}(t) \\
r_{2 n+1}(t) \\
\vdots \\
r_{2 j}(t) \\
r_{2 j+1}(t) \\
\vdots
\end{array}\right)
$$


and

$$
A_{s t}=\left(\begin{array}{cccc}
A_{n} & 0 & \cdots & \\
0 & \ddots & 0 & \\
\vdots & 0 & A_{j} & \ddots \\
& & \ddots & \ddots
\end{array}\right) \quad \text { and } \quad B_{s t}=\left(\begin{array}{c}
B_{n} \\
\vdots \\
B_{j} \\
\vdots
\end{array}\right)
$$

Note that

$$
r_{u n}(t)=\sum_{j=0}^{2 n-1} r_{j}(t) \tilde{e}_{j}, \quad r_{s t}(t)=\sum_{j=2 n}^{+\infty} r_{j}(t) \tilde{e}_{j} .
$$

Stabilization of the unstable finite-dimensional part. Let us analyze the finite-dimensional linear autonomous control system (3.3).

Lemma 3.7. Let $a \in(0, L)$. Assume that $d_{3} \neq 0$. There exists a finite discrete set $\mathcal{Z} \subset(0, L)$ such that, for every $b$ in $(0, L) \backslash \mathcal{Z}$ such that $a<b$, the pair $\left(A_{\text {un }}, B_{\text {un }}\right)$ satisfies the Kalman condition, that is,

$$
\operatorname{rank}\left(B_{u n}, A_{u n} B_{u n}, \ldots, A_{u n}^{2 n-1} B_{u n}\right)=2 n .
$$

Proof. Let us diagonalize $A_{\text {un }}$ in $\mathbb{C}$ by writing $A_{\text {un }}=Q D Q^{-1}$ with $Q$ an invertible complex matrix of size $2 n$ and $D$ a diagonal matrix of size $2 n$ whose diagonal is $\left(\mu_{0}, \nu_{0}, \ldots, \mu_{n-1}, \nu_{n-1}\right)$, where the pair $\left(\mu_{j}, \nu_{j}\right)$ is the spectrum of $A_{j}$ for every $j$. In other words, $\mu_{j}$ and $\nu_{j}$ are the two roots of the polynomial

$$
\pi_{A_{j}}(X)=X^{2}-\tau_{j} X+\delta_{j},
$$

where $\tau_{j}=2 \lambda_{j}+1+E_{n}$ and $\delta_{j}=2\left(\lambda_{j}+1\right)\left(\lambda_{j}+E_{n}\right)$. In particular, since $\lambda_{0}=-E_{n}$, we have $\mu_{0}=0, \nu_{0}=1-E_{n}$.

Noting that

$$
\begin{aligned}
& \operatorname{rank}\left(B_{u n}, A_{u n} B_{u n}, \ldots, A_{u n}^{2 n-1} B_{x u n}\right) \\
= & \operatorname{rank}\left(Q^{-1} B_{u n}, D Q^{-1} B_{u n}, \ldots, D^{2 n-1} B_{u n}\right),
\end{aligned}
$$

and setting

$$
Q^{-1} B_{u n}=\left(\begin{array}{c}
\beta_{1} \\
\vdots \\
\beta_{2 n}
\end{array}\right)
$$

we easily compute

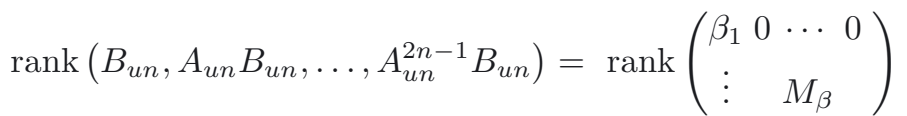

$$
\begin{aligned}
& =\operatorname{rank}\left(\begin{array}{cccc}
\beta_{1} & 0 & \cdots & 0 \\
\beta_{2} & \nu_{0} \beta_{2} & \cdots & \nu_{0}^{2 n-1} \beta_{2} \\
\beta_{3} & \mu_{1} \beta_{3} & \cdots & \mu_{1}^{2 n-1} \beta_{3} \\
\vdots & \vdots & & \vdots \\
\beta_{2 n-1} & \mu_{n-1} \beta_{2 n-1} & \cdots & \mu_{n-1}^{2 n-1} \beta_{2 n-1} \\
\beta_{2 n} & \nu_{n-1} \beta_{2 n} & \cdots & \nu_{n-1}^{2 n-1} \beta_{2 n}
\end{array}\right) .
\end{aligned}
$$

Identifying Vandermonde matrices inside the above matrix, we infer that this rank is equal to $2 n$ if and only if the following three conditions are satisfied:

C1. $\nu_{0}, \mu_{1}, \nu_{1}, \mu_{2}, \nu_{2}, \ldots, \mu_{n-2}, \nu_{n-2}, \mu_{n-1}, \nu_{n-1}$ are all distinct; 
C2. $\beta_{k} \neq 0$ for every $k \in\{2, \ldots, 2 n\}$;

C3. $\beta_{1} \neq 0$.

The end of the proof is devoted to prove that $\mathbf{C 1}, \mathbf{C} 2$ and $\mathbf{C 3}$ hold under the generic assumptions announced in the statement of the lemma.

Condition C1. Fix $j \in\{0, \cdots, n-1\}$ and recall that $\lambda_{j} \in(-1,0)$. Let us first prove that the roots of the polynomial $\pi_{A_{j}}$ are distinct. It is easy to establish that

$$
\sup _{x \in[-1,0]}\left(x^{2}+\left(E_{n}+1\right) x-\left(E_{n}-1\right)^{2}\right) \leqslant-\left(E_{n}-1\right)^{2},
$$

and therefore the equation $\lambda_{j}^{2}+\left(1+E_{n}\right) \lambda_{j}-\left(E_{n}-1\right)^{2}=0$ cannot hold. In other words, the equation $\operatorname{tr}\left(A_{j}\right)^{2}=$ $4 \operatorname{det}\left(A_{j}\right)^{2}$ cannot hold.

It remains to prove that the roots of the polynomials $\pi_{A_{k}}$ are all pairwise distinct for $k \in\{1, \cdots, n-1\}$. To prove that fact, we consider the resultant ${ }^{4} \operatorname{Res}\left(\pi_{A_{j}}, \pi_{A_{k}}\right)$ of $\pi_{A_{j}}$ and $\pi_{A_{k}}$. We compute

$$
\operatorname{Res}\left(\pi_{\mathcal{A}_{j}}, \pi_{\mathcal{A}_{k}}\right)=\operatorname{det}\left(\begin{array}{cccc}
1 & 0 & 1 & 0 \\
-\tau_{k} & 1 & -\tau_{j} & 1 \\
\delta_{k} & -\tau_{k} & \delta_{j} & -\tau_{j} \\
0 & \delta_{k} & 0 & \delta_{j}
\end{array}\right)=2\left(\lambda_{j}-\lambda_{k}\right)^{2}\left(\delta_{j}+\delta_{k}\right),
$$

and hence $\operatorname{Res}\left(\pi_{\mathcal{A}_{j}}, \pi_{\mathcal{A}_{k}}\right) \neq 0$ for all $j, k \in\{1, \cdots, n-1\}$. Hence $\mathbf{C} \mathbf{1}$ is true.

Condition C2. We compute explicitly

$$
Q^{-1}=\left(\begin{array}{ccc}
Q_{0}^{-1} & & 0 \\
& \ddots & \\
0 & & Q_{n-1}^{-1}
\end{array}\right)
$$

where

$$
Q_{0}^{-1}=\frac{1}{1-E_{n}}\left(\begin{array}{cc}
1 & 1 \\
-1 & 0
\end{array}\right)
$$

and

$$
Q_{k}^{-1}=-\frac{1}{\left(\lambda_{k}+E_{n}\right)\left(\nu_{k}-\mu_{k}\right)}\left(\begin{array}{cc}
\lambda_{k}-\nu_{k} & -\left(\lambda_{k}+E_{n}\right) \\
\mu_{k}-\left(\lambda_{k}+1\right) & \lambda_{k}+E_{n}
\end{array}\right)
$$

for every $k \in\{1, \cdots, n-1\}$. Now, the condition $\mathbf{C 2}$ is not satisfied if and only if

$$
\prod_{i=2}^{2 n} \beta_{i}=0
$$

This equation yields an analytic constraint equation in $b$. Since the set of zeros of an analytic function over a compact interval is finite, we infer that, for all values of $b$ such that $a<b$ but maybe a finite number of them, the condition $\mathbf{C 2}$ is satisfied.

Condition C3. Easy computations show that

$$
\beta_{1}=\frac{2 d_{3}}{1-E_{n}} \int_{a}^{b} e_{1}(x) \mathrm{d} x
$$

As previously, the quantity $\int_{a}^{b} e_{1}(x) \mathrm{d} x$ do not vanish except maybe for a finite number of values of $b$ and then the condition $\mathbf{C} 3$ is satisfied.

This finishes the proof of the lemma.

\footnotetext{
${ }^{4}$ Recall that the resultant of two polynomials is a polynomial expression of their coefficients, which is equal to zero if and only if the polynomials have a common root.
} 
Remark 3.8. The formula (3.7) shows that, if the axis of the solenoid is parallel to the nanowire, that is, if $d_{3}=0$, then $\beta_{1}=0$. Then the Kalman condition is never satisfied in this case. This justifies the contents of Remark 3.5.

Remark 3.9. Let us stress once again the importance of the fact that -1 is not an eigenvalue of $A$ (see Prop. 2.3). Indeed if -1 were to be an eigenvalue, then the conclusion of Lemma 3.7 would not hold true anymore since the first and last rows of the Kalman matrix

$$
\left(B_{u n}, A_{u n} B_{u n}, \ldots, A_{u n}^{2 n-1} B_{u n}\right)
$$

would be collinear. In such a case, it would have been necessary to have two solenoids (and hence two controls) instead of a single one in order to perform the same analysis and to get the same kinds of results.

Since the control system (3.3) satisfies the Kalman condition, we get the following corollary, as a consequence of the pole-shifting theorem (see, e.g., $[18,26])$.

Corollary 3.10. In the conditions of Lemma 3.7, there exists a $2 \times 2 n$ matrix $K$ such that the matrix $M=$ $A_{u n}+B_{u n} K$ is Hurwitz, and there exists a symmetric positive definite matrix $P$ of size $2 n$ such that

$$
P^{\top} M+M P=-I_{2 n} .
$$

In particular this means that the feedback control

$$
u(t)=K r_{u n}(t)
$$

stabilizes the finite-dimensional control system (3.3). Moreover, the functional

$$
V_{u n}\left(r_{u n}\right)=\frac{1}{2} r_{u n}^{\top} P r_{u n}
$$

is a Lyapunov function for the closed-loop system

$$
\dot{r}_{u n}(t)=\left(A_{u n}+B_{u n} K\right) r_{u n}(t) .
$$

Stabilization of the whole system. Let us prove that the feedback (3.9) stabilizes locally the whole infinitedimensional system (2.19) with the remainder term $R$.

Using the notations introduced previously, $r_{u n}$ and $r_{s t}$ are solutions of the infinite-dimensional system

$$
\begin{aligned}
\dot{r}_{u n} & =\left(A_{u n}+B_{u n} K\right) r_{u n}+R_{1}\left(x, r, r_{x}, r_{x x}, u\right), \\
\dot{r}_{s t} & =A_{s t} r_{s t}+B_{s t} K r_{u n}+R_{2}\left(x, r, r_{x}, r_{x x}, u\right),
\end{aligned}
$$

where

$$
R=\left(\begin{array}{l}
R_{1} \\
R_{2}
\end{array}\right)
$$

In order to prove the local asymptotic stability of this system yielding the desired stability property in $H^{1}$ topology, we are going to design an appropriate Lyapunov function.

First of all, since the $2 \times 2$ matrix $A_{j}$ are Hurwitz for every $j \geqslant n$ (see Lem. 2.6), and therefore, as in Corollary 3.10, it follows from the well-known Lyapunov lemma that there exists a symmetric positive definite matrix $Q_{j}$ of size 2 such that

$$
A_{j}^{\top} Q_{j}+Q_{j} A_{j}=\lambda_{j} I_{2} .
$$

Note the important fact that $\lambda_{j}$ appears at the right-hand side of (3.11), with $\lambda_{j} \rightarrow-\infty$ as $j$ tends to $+\infty$. The term $\lambda_{j}$ is a weight that will be important to get the stability property in $H^{1}$ topology, combined with the following also instrumental lemma. 
Lemma 3.11. The matrices $Q_{j}$ are uniformly bounded.

Proof. Clearly the matrix

$$
Q_{j}=\lambda_{j} \int_{0}^{+\infty} \mathrm{e}^{t A_{j}^{\top}} \mathrm{e}^{t A_{j}} \mathrm{~d} t
$$

is a solution of the Lyapunov equation (3.11), and it easily follows from the structure of the matrices $A_{j}$ and from the fact that $\lambda_{j} \rightarrow-\infty$ as $j$ tends to $+\infty$ that there exists $C>0$ such that

$$
\mathrm{e}^{t A_{j}} \leqslant C \mathrm{e}^{\lambda_{j} t}
$$

for every $t \geqslant 0$ and for every $j \in \mathbb{N}$. Therefore

$$
\left\|Q_{j}\right\| \leqslant C\left|\lambda_{j}\right| \int_{0}^{+\infty} \mathrm{e}^{2 \lambda_{j} t} \mathrm{~d} t=\frac{C}{2}
$$

as desired.

We define the infinite-dimensional matrix

$$
Q^{\lambda}=\left(\begin{array}{cccc}
\lambda_{n} Q_{n} & 0 & \ldots & \\
0 & \ddots & 0 & \\
\vdots & 0 & \lambda_{j} Q_{j} & \ddots \\
& & \ddots & \ddots
\end{array}\right),
$$

and we define the functional

$$
\begin{aligned}
\mathcal{V}(r) & =\frac{c}{2} r_{u n}^{\top} P r_{u n}+\frac{1}{2} r_{s t}^{\top} Q^{\lambda} r_{s t} \\
& =\frac{c}{2} r_{u n}^{\top} P r_{u n}+\frac{1}{2} \sum_{j=n}^{+\infty}\left|\lambda_{j}\right|\left(r_{2 j} r_{2 j+1}\right) Q_{j}\left(\begin{array}{c}
r_{2 j} \\
r_{2 j+1}
\end{array}\right)
\end{aligned}
$$

for every $r \in H^{1}\left(0, L ; \mathbb{R}^{2}\right)$, where $c>0$ will be chosen large enough.

Lemma 3.12. There exist $C_{1}>0$ and $C_{2}>0$ such that

$$
C_{1}\|r\|_{H^{1}\left(0, L ; \mathbf{R}^{2}\right)}^{2} \leqslant \mathcal{V}(r) \leqslant C_{2}\|r\|_{H^{1}\left(0, L ; \mathbb{R}^{2}\right)}^{2},
$$

for every $r \in H^{1}\left(0, L ; \mathbb{R}^{2}\right)$. In other words the norm $\sqrt{\mathcal{V}(r)}$ is equivalent to the $H^{1}$ norm.

Proof. We denote by $\sim$ the equivalence of norms. Since $P$ is symmetric positive definite, it is clear that

$$
\left(r_{u n}^{\top} P r_{u n}\right)^{1 / 2} \sim\left\|r_{u n}\right\|=\left(\sum_{j=0}^{2 n-1} r_{j}^{2}\right)^{1 / 2}
$$

in the finite-dimensional space $\mathbb{R}^{2 n}$. Besides, by definition of the operator $A$, one has

$$
\langle-A f, f\rangle_{L^{2}(0, L)}=\int_{0}^{L}\left(f^{\prime}(x)^{2}+2 \cos ^{2} \theta(x) f(x)^{2}\right) \mathrm{d} x
$$


and then clearly

$$
\left(\langle-A f, f\rangle_{L^{2}(0, L)}\right)^{1 / 2} \sim\|f\|_{H^{1}(0, L)}
$$

It follows easily that

$$
\|r\|_{H^{1}\left(0, L ; \mathbb{R}^{2}\right)} \sim\left(\sum_{j=0}^{+\infty}\left|\lambda_{j}\right|\left(r_{2 j}^{2}+r_{2 j+1}^{2}\right)\right)^{1 / 2}
$$

Therefore it suffices to prove that

$$
\left(\sum_{j=n}^{+\infty}\left|\lambda_{j}\right|\left(r_{2 j} r_{2 j+1}\right) Q_{j}\left(\begin{array}{c}
r_{2 j} \\
r_{2 j+1}
\end{array}\right)\right)^{1 / 2} \sim\left(\sum_{j=n}^{+\infty}\left|\lambda_{j}\right|\left(r_{2 j}^{2}+r_{2 j+1}^{2}\right)\right)^{1 / 2} .
$$

But this follows from the fact that the matrices $Q_{j}$ are uniformly bounded.

Remark 3.13. Note that, similarly,

$$
\|r\|_{H^{2}\left(0, L ; \mathbb{R}^{2}\right)} \sim\left(\sum_{j=0}^{+\infty} \lambda_{j}^{2}\left(r_{2 j}^{2}+r_{2 j+1}^{2}\right)\right)^{1 / 2} .
$$

Let $r$ be a solution of (3.10). Using (3.8) and (3.11), we compute

$$
\begin{aligned}
\frac{\mathrm{d}}{\mathrm{d} t} \mathcal{V}(r(t, \cdot))= & -c\left\|r_{u n}(t)\right\|_{\mathbf{R}^{2 n)}}^{2}-\sum_{j=n}^{+\infty} \lambda_{j}^{2}\left(r_{2 j}^{2}+r_{2 j+1}^{2}\right) \\
& +r_{u n}^{\top} P R_{1}+r_{s t}^{\top} Q^{\lambda} B_{s t} K r_{u n}+r_{s t}^{\top} Q^{\lambda} R_{2}
\end{aligned}
$$

We have

$$
\left\|Q^{\lambda} r_{s t}\right\|^{2} \leqslant C_{3} \sum_{j=n}^{+\infty}\left|\lambda_{j}\right|\left(r_{2 j}^{2}+r_{2 j+1}^{2}\right)
$$

for some constant $C_{3}>0$, and hence using Young's inequality and the fact that $\left|\lambda_{j}\right| \leqslant \lambda_{j}^{2}$ as $j$ tends to $+\infty$ it follows that there exists $C_{4}>0$ such that

$$
\left|r_{s t}^{\top} Q^{\lambda} B_{s t} K r_{u n}\right| \leqslant C_{4}\left\|r_{u n}(t)\right\|_{\mathbb{R}^{2 n)}}^{2}+\frac{1}{2} \sum_{j=n}^{+\infty} \lambda_{j}^{2}\left(r_{2 j}^{2}+r_{2 j+1}^{2}\right) .
$$

Now, choosing $c>0$ large enough (for instance, $c>2 C_{4}$ ), we infer from Remark 3.13, from (3.15) and (3.17) that there exists $C_{5}>0$ such that

$$
\begin{aligned}
\frac{\mathrm{d}}{\mathrm{d} t} \mathcal{V}(r(t, \cdot)) \leqslant & -C_{5}\|r(t, \cdot)\|_{H^{1}\left(0, L ; \mathbb{R}^{2}\right)}^{2}-C_{5}\|r(t, \cdot)\|_{H^{2}\left(0, L ; \mathbb{R}^{2}\right)}^{2} \\
& +C_{5}\|r(t, \cdot)\|_{H^{1}\left(0, L ; \mathbb{R}^{2}\right)}\|R\|_{L^{2}\left(0, L ; \mathbb{R}^{2}\right)} .
\end{aligned}
$$


It follows from the estimate $(2.23)$ of the remainder term $R$ that

$$
\begin{aligned}
\|r(t, \cdot)\|_{H^{1}\left(0, L ; \mathbb{R}^{2}\right)}\|R\|_{L^{2}\left(0, L ; \mathbb{R}^{2}\right)} \leqslant & C\left(\|r(t, \cdot)\|_{L^{2}\left(0, L ; \mathbb{R}^{2}\right)}^{2}\|r(t, \cdot)\|_{H^{1}\left(0, L ; \mathbb{R}^{2}\right)}\|r(t, \cdot)\|_{H^{2}\left(0, L ; \mathbb{R}^{2}\right)}\right. \\
& +\|r(t, \cdot)\|_{L^{2}\left(0, L ; \mathbb{R}^{2}\right)}\|r(t, \cdot)\|_{H^{1}\left(0, L ; \mathbb{R}^{2}\right)}^{2} \\
& +\|r(t, \cdot)\|_{L^{2}\left(0, L ; \mathbb{R}^{2}\right)}\|r(t, \cdot)\|_{H^{1}\left(0, L ; \mathbb{R}^{2}\right)}^{3} \\
& \left.+\|r(t, \cdot)\|_{L^{2}\left(0, L ; \mathbb{R}^{2}\right)}^{2}\|r(t, \cdot)\|_{H^{1}\left(0, L ; \mathbb{R}^{2}\right)}\right) \\
\leqslant & C\left(\|r(t, \cdot)\|_{H^{1}\left(0, L ; \mathbb{R}^{2}\right)}^{3}\|r(t, \cdot)\|_{H^{2}\left(0, L ; \mathbb{R}^{2}\right)}\right. \\
& \left.+2\|r(t, \cdot)\|_{H^{1}\left(0, L ; \mathbb{R}^{2}\right)}^{3}+\|r(t, \cdot)\|_{H^{1}\left(0, L ; \mathbb{R}^{2}\right)}^{4}\right) .
\end{aligned}
$$

Here, we need an a priori argument: as long as $\mathcal{V}(r(t, \cdot))$ remains small enough, or equivalently as long as $\|r(t, \cdot)\|_{H^{1}\left(0, L ; \mathbb{R}^{2}\right)}$ remains small enough (which will be verified a posteriori), we can write

$$
\|r(t, \cdot)\|_{H^{1}\left(0, L ; \mathbb{R}^{2}\right)}^{2} \leqslant\|r(t, \cdot)\|_{H^{1}\left(0, L ; \mathbb{R}^{2}\right)},
$$

and hence, using again Young's inequality, it follows that there exists $C_{6}>0$ such that

$$
C_{5}\|r(t, \cdot)\|_{H^{1}\left(0, L ; \mathbb{R}^{2}\right)}\|R\|_{L^{2}\left(0, L ; \mathbb{R}^{2}\right)} \leqslant \frac{C_{5}}{2}\|r(t, \cdot)\|_{H^{2}\left(0, L ; \mathbb{R}^{2}\right)}^{2}+C_{6} \sqrt{\mathcal{V}(r(t, \cdot))}\|r(t, \cdot)\|_{H^{1}\left(0, L ; \mathbb{R}^{2}\right)}^{2} .
$$

Therefore, from (3.18) and (3.19) we infer that

$$
\begin{aligned}
\frac{\mathrm{d}}{\mathrm{d} t} \mathcal{V}(r(t, \cdot)) & \leqslant-\left(C_{5}-C_{6} \sqrt{\mathcal{V}(r(t, \cdot))}\right)\|r(t, \cdot)\|_{H^{1}\left(0, L ; \mathbb{R}^{2}\right)}^{2}-\frac{C_{5}}{2}\|r(t, \cdot)\|_{H^{2}\left(0, L ; \mathbb{R}^{2}\right)}^{2} \\
& \leqslant-\left(C_{5}-C_{6} \sqrt{\mathcal{V}(r(t, \cdot))}\right)\|r(t, \cdot)\|_{H^{1}\left(0, L ; \mathbb{R}^{2}\right)}^{2},
\end{aligned}
$$

and hence, as long as $\mathcal{V}(r(t, \cdot))$ remains small enough (here, we need for instance the a priori estimate $\left.C_{6} \sqrt{\mathcal{V}(r(t, \cdot))}<C_{5} / 2\right)$, we have, using Lemma 3.12,

$$
\frac{\mathrm{d}}{\mathrm{d} t} \mathcal{V}(r(t, \cdot)) \leqslant-C_{7} \mathcal{V}(r(t, \cdot))
$$

It follows that $\mathcal{V}(r(t, \cdot))$ decreases exponentially. This shows that the above a priori estimates are valid, provided that $\mathcal{V}(r(0, \cdot))$ is small enough.

This proves the local stability result in $H^{1}$ norm claimed in Theorem 3.1.

Sketch of proof of Theorem 3.6. Considering $\bar{m}^{1}$ and $\bar{m}^{2}$ as in the statement of Theorem 3.6, it is clear that

$$
\bar{m}(\tau, x)=\left(\begin{array}{c}
\cos \theta(x) \\
\cos \left((1-\tau) \omega_{1}+\tau \omega_{2}\right) \sin \theta(x) \\
\sin \left((1-\tau) \omega_{1}+\tau \omega_{2}\right) \sin \theta(x)
\end{array}\right),
$$

with $0 \leqslant \tau \leqslant 1$, yields a path of steady-states joining $\bar{m}^{1}$ to $\bar{m}^{2}$. The strategy developed in $[11,12]$ consists of tracking the path $\bar{m}(\varepsilon t, \cdot)$ for some $\varepsilon>0$ small enough. The role of $\varepsilon$ is to slow down the time so that, when linearizing (1.6) along the path, we get a slowly-varying in time linear control system.

We do not give all details since the proof is then very similar to the one written previously. We only underline the slight differences in every step. 
The first step consists of writing the linearized system. The resulting linear system is still of the form (3.1). Note that, since every steady-state $\bar{m}(\tau, \cdot)$ is the image of $M_{0}$ by the rotation with axis $e_{1}$ and angle $\omega$, the linearized system around $\bar{m}(\tau, \cdot)$ is as well obtained by rotation of the linearized system around $M_{0}$. The spectral properties are the same.

An important point to be underlined is the following, and here appears the crucial role of $\varepsilon$. The resulting finite-dimensional system, containing all unstable modes, is of the form

$$
\dot{Y}(t)=A_{u n}(\varepsilon t) Y(t)+B_{u n}(\varepsilon t) u(t)
$$

and the Kalman condition holds for the pair $\left(A_{u n}(\tau), B_{u n}(\tau)\right)$ for every $\tau \in[0,1]$. If $\varepsilon$ were taken equal to 1 , then the Kalman condition could fail to imply the desired stabilization result (as is well-known, see [18]). This implication is however recovered for slowly-varying in time linear systems. Hence if one takes $\varepsilon>0$ small enough then we get a statement similar to Corollary 3.10, with matrices that depend on $\varepsilon t$. The feedback that we design is therefore of the form $u(t)=K(\varepsilon t) r_{u n}(t)$.

Then the stabilization of the whole system follows the same lines as before, with the difference that the equations (3.10) involve one additional remainder term, which is of the order of $\varepsilon$ and which comes from the derivative in time of $\bar{m}(\varepsilon t, \cdot)$. This additional term is then recovered at the right-hand side (3.20), and this means that $\mathcal{V}(r(t, \cdot))$ may not decrease exponentially but anyway we get that $\mathcal{V}(r(1 / \varepsilon, \cdot))$ is of the order of $\varepsilon$, which yields the desired result.

\section{REFERENCES}

[1] D.A. Allwood et al., Submicrometer ferromagnetic NOT gate and shift register. Science 296 (2002) $2003-2006$.

[2] F. Alouges and A. Soyeur, On global weak solutions for Landau-Lifshitz equations: existence and nonuniqueness. Nonlinear Anal. 18 (1992) 1070-1084.

[3] D. Atkinson et al., Magnetic domain-wall dynamics in a submicrometre ferromagnetic structure. Nature Mater. 2 (2003) 85-87.

[4] G.S.D. Beach, C. Nistor, C. Knutson, M. Tsoi and J.L. Erskine, Dynamics of field-driven domain-wall propagation in ferromagnetic nanowires. Nature Mater. 4 (2005) 741-744.

[5] F. Brown, Micromagnetics. Wiley, New York (1963).

[6] G. Carbou and S. Labbé, Stability for walls in ferromagnetic nanowire. Discrete Contin. Dyn. Syst. Ser. B 6 (2006) 273-290.

[7] G. Carbou and P. Fabrie, Regular solutions for Landau-Lifshitz equation in $\mathbb{R}^{3}$. Commun. Appl. Anal. 5 (2001) 17-30.

[8] G. Carbou and S. Labbé, Stabilization of walls for nanowires of finite length. ESAIM: COCV 18 (2012) 1-21.

[9] G. Carbou, S. Labbé and E. Trélat, Control of travelling walls in a ferromagnetic nanowire. Discrete Contin. Dyn. Syst. Ser. $S 1$ (2008) 51-59.

[10] G. Carbou, S. Labbé and E. Trélat, Smooth control of nanowires by means of a magnetic field. Commun. Pure Appl. Anal. 8 (2009) 871-879.

[11] J.-M. Coron and E. Trélat, Global steady-state controllability of 1-D semilinear heat equations. SIAM J. Control Optim. 43 (2004) 549-569.

[12] J.-M. Coron and E. Trélat, Global steady-state stabilization and controllability of 1-D semilinear wave equations. Commun. Contemp. Math. 8 (2006) 535-567.

[13] A. De Simone, H. Knüpfer and F. Otto, $2-d$ stability of the Néel wall. Calc. Var. Partial Differ. Equ. 27 (2006) $233-253$.

[14] Y. Egorov and V. Kondratiev, On spectral theory of elliptic operators. Birkhäuser (1996).

[15] J. Grollier et al., Switching a spin valve back and forth by current-induced domain wall motion. Appl. Phys. Lett. 83 (2003) $509-511$.

[16] A. Hubert and R. Schäfer, Magnetic domains: the analysis of magnetic microstructures. Springer-Verlag (2000).

[17] R. Ignat and B. Merlet, Lower bound for the energy of Bloch Walls in micromagnetics. Arch. Ration. Mech. Anal. 199 (2011) $369-406$.

[18] H.K. Khalil, Nonlinear Systems. Macmillan Publishing Company, New York (1992).

[19] S. Labbé, Y. Privat and E. Trélat, Stability properties of steady-states for a network of ferromagnetic nanowires. J. Differ. Equ. 253 (2012) 1709-1728.

[20] L. Landau and E. Lifshitz, Electrodynamics of continuous media, Course of theoretical Physics. Vol. 8. Translated from the russian by J.B. Sykes and J.S. Bell. Pergamon Press, Oxford-London-New York-Paris, Addison-Wesley Publishing Co., Inc., Reading, Mass (1960).

[21] C. Melcher, Global solvability of the Cauchy problem for the Landau-Lifshitz-Gilbert equation in higher dimensions. Indiana University Math. J. 61 (2013) 1175-1200.

[22] C. Melcher and M. Ptashnyk, Landau-Lifshitz-Slonczewski equations: global weak and classical solutions. SIAM J. Math. Anal. 45 (2013) 407-429. 
[23] T. Ono et al., Propagation of a domain wall in a submicrometer magnetic wire. Science 284 (1999) 468-470.

[24] S. Parkin et al., Magnetic domain-wall racetrack memory. Science 320 (2008) 190-194.

[25] D. Sanchez, Behaviour of the Landau-Lifshitz equation in a periodic thin layer. Asymptot. Anal. 41 (2005) 41-69.

[26] E. Trélat, Contrôle optimal (French) [Optimal control], Théorie \& applications [Theory and applications]. Math. Concrètes [Concrete Mathematics]. Vuibert, Paris (2005).

[27] M. Tsoi, R.E. Fontana and S.S.P. Parkin, Magnetic domain wall motion triggered by an electric current. Appl. Phys. Lett. 83 (2003) 2617-2619.

[28] A. Visintin, On Landau-Lifshitz equations for ferromagnetism. Japan J. Appl. Math. 2 (1985) 69-84.

[29] A. Zettl, Sturm-Liouville theory. Vol. 121 of Math. Surveys $\& 3$ Monographs. AMS, Providence (2005). 